\title{
HUBBLE SPACE TELESCOPE IMAGES OF FOUR MAGELLANIC CLOUD PLANETARY NEBULAE
}

\author{
M. J. BARLOW \\ Department of Physics \& Astronomy, University College London, \\ Gower Street, London WC1E 6BT, U.K. \\ and \\ J. C. BLADES, S. OSMER \& THE FAINT OBJECT CAMERA I.D.T. \\ Space Telescope Science Institute, \\ 3700 San Martin Drive, Baltimore, MD 21218, USA
}

\begin{abstract}
Using the Faint Object Camera on-board the Hubble Space Telescope, we have obtained images of four planetary nebulae in the Magellanic Clouds, namely N2 and N5 in the SMC and N66 and N201 in the LMC. Each nebula was imaged through narrow-band filters isolating [O III] $\lambda 5007$ and $H \beta$, for a nominal exposure time of 1000 seconds in each filter. The f/96 optical chain of the FOC was used, yielding $512 \times 5120.022$ arcsec square pixels. Considerable detail is evident on the raw images and after deconvolution using the Richardson-Lucy algorithm, structures as small as 0.06 arcsec are easily discernible. Figure 1 shows NS and EW intensity cross-cuts through the deconvolved [O III] $\lambda 5007$ images of SMC N2 and SMC N5. SMC N2 is a slightly elleptical ringlike nebula, with its greatest elongation in the EW direction. The peak-to-peak dimensions of the ring are $0.21 \times 0.26 \operatorname{arcsec}^{2}$. SMC N5 has a circular ring shape, with the [O III] $\lambda 5007$ image showing a clearly defined, nearly uniform structure, apart from a bright patch at the northern edge. The peak-to-peak diameter of the ring is 0.26 arcsec and the ring itself is significantly narrower than that of SMC N2, with a width as small as 0.06 arcsec (FWHM) in some places. LMC N201 is very compact, with a FWHM of 0.21 arcsec in the H $\beta$ image. The Type I PN LMC N66 is a multi-polar nebula, with the brightest part having an extent of about 2 arcsec. Its structure is extremely complex, with several bright knots and faint loops visible outside the two bright lobes. A full description of our results can be found in Blades et al., ApJ, 398, L41-44.
\end{abstract}

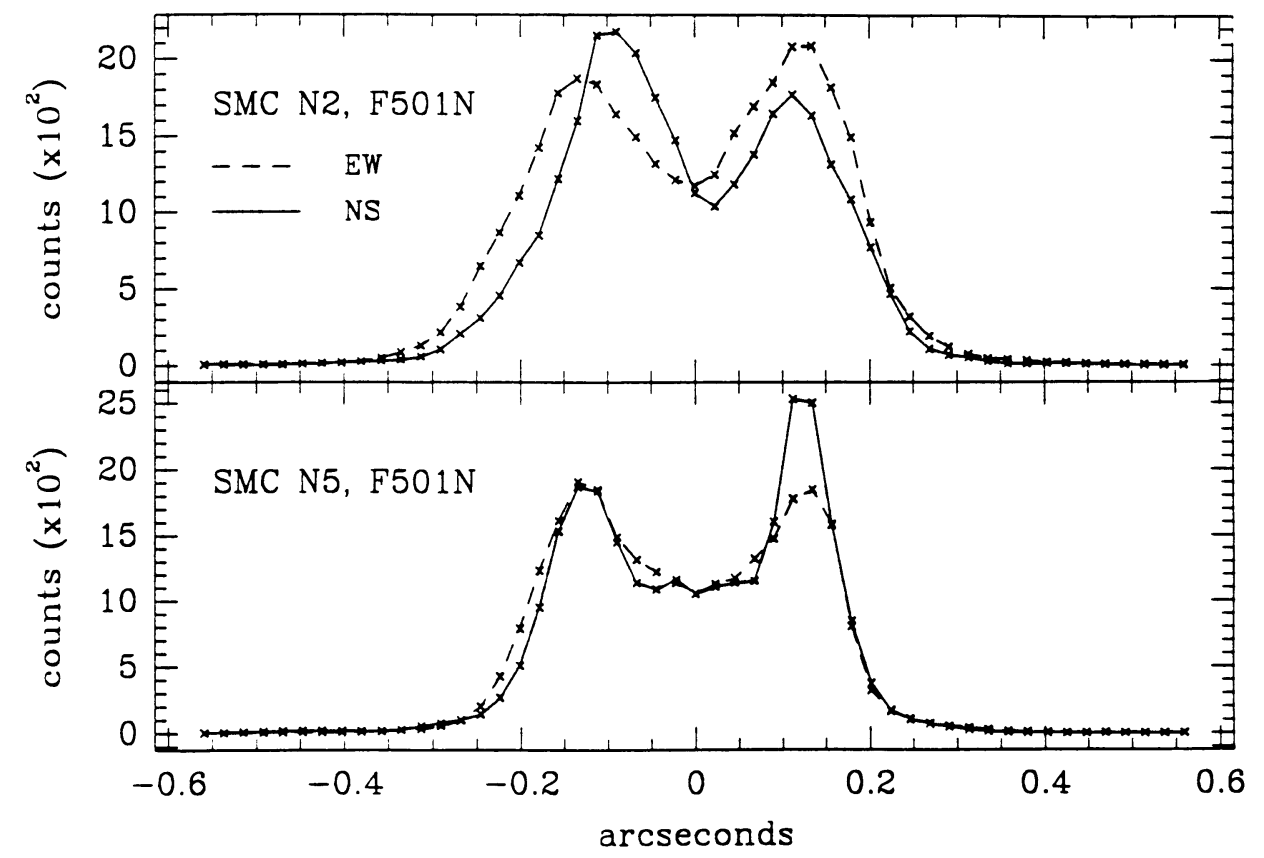

